\title{
$g$-factors and discrete energy level velocities in nanoparticles
}

\author{
Eduardo R. Mucciolo, ${ }^{1}$ Caio H. Lewenkopf, ${ }^{2}$ and Leonid I. Glazman ${ }^{3}$ \\ ${ }^{1}$ Department of Physics, University of Central Florida, \\ P.O. Box 162385, Orlando, Florida 32816-2385, USA \\ ${ }^{2}$ Instituto de Física, Universidade do Estado do Rio de Janeiro, \\ R. São Francisco Xavier 524, 20550-900 Rio de Janeiro, Brazil, and \\ ${ }^{3}$ Theoretical Physics Institute, University of Minnesota, Minneapolis, Minnesota 55455, USA
}

(Dated: February 9, 2019)

\begin{abstract}
We establish relations between the statistics of $g$ factors and the fluctuations of energy in metallic nanoparticles where spin-orbit coupling is present. These relations assume that the electron dynamics in the grain is chaotic. The expressions we provide connect the second moment of the $g$ factor to the root-mean square "level velocity" (the derivative of the energy with respect to magnetic field) calculated at magnetic fields larger than a characteristic correlation field. Our predictions relate readily observable quantities and allow for a parameter-free comparison with experiments.
\end{abstract}

PACS numbers: 73.23.Hk, 71.70.Ej

It was noted in experiments ${ }^{1.2}$ that the Zeeman splitting of discrete energy levels in nanoparticles is very sensitive to the presence of spin-orbit interaction. The splitting can be characterized by a level-dependent $g$ factor. Adding to the $\mathrm{Al}$ grains only $4 \%$ of $\mathrm{Au}$ resulted in a change of measured $g$ factors from approximately 1.7 to 0.7 . In addition to being suppressed, the $g$ factor in the presence of spin-orbit interaction also fluctuates randomly from level to level.

These fluctuations of $g$ factors were described in the framework of random matrix theory ${ }^{3.4 .5}$ (RMT) and the suppression of the $g$ factor was related to the strength of the spin-orbit interaction and to the elastic mean free path of electrons in the grains $\underline{\underline{4}}$ The limit of strong spinorbit interaction corresponds to a short spin-orbit scattering time, $\tau_{\text {so }} \delta / \hbar \ll 1$, where $\delta^{-1}$ is the mean density of states in the grain at zero field. It was predicted that the distribution of eigenvalues of the $g$-factor tensor in this limit should have a Gaussian form. Within RMT, this distribution is characterized by a phenomenological parameter $\left\langle g^{2}\right\rangle$. In Ref. 4, this parameter was expressed in terms of the grain size, electron mean free path $l$, and relaxation time $\tau_{\text {so }}$. However, a comparison of the experimental results with theory was not entirely satisfactory. On the one hand, there is an indication that the distribution of the eigenvalues and directions of the eigenvectors of the $g$-tensor is Gaussian and correspond to a "pure" symplectic ensemble. 6.7 On the other hand, it is not clear ${ }^{6}$ whether the small values of $\left\langle g^{2}\right\rangle$ obtained in the experiments agree with the theoretical values estimated in Ref. 4. The difficulty in making the comparison comes from the lack of information about the amount of disorder in the grain.

The goal of this work is to provide three relations for the distribution width $\left\langle g^{2}\right\rangle$ to other quantities that are directly measured in the same set of experiments (and thus do not rely on any additional information about the amount of disorder in the grains). These quantities are the variance of the energy level derivative with respect to the magnetic field (known as level velocity ${ }^{8}$ ) and the zero-magnetic field level curvature (the second derivative of the energy level with respect to magnetic field).

We begin by stating our main results. Our first expression, valid for strong spin-orbit coupling only, $\tau_{\text {so }} \delta / \hbar \ll$ 1 , is

$$
\left\langle g^{2}\right\rangle=\frac{12}{\mu_{B}^{2}} \operatorname{var}\left[\left(\frac{d \varepsilon_{\nu}}{d B}\right)_{B \gg B^{*}}\right]_{\tau_{\mathrm{so}} \rightarrow 0},
$$

where var[] denotes the variance, $\mu_{B}$ is the Bohr magneton, and $B^{*}$ is the crossover field for breaking timereversal symmetry. Equation (11) gives a statistical connection between the response at $B \rightarrow 0$ (the $g$ factor) to that at large magnetic fields (the level velocity). It provides a way to check experimentally if the grains exhibiting fluctuations of the $g$ factor indeed belong to a "pure" symplectic ensemble, rather than to an ensemble describing the crossover between the orthogonal and symplectic limits. Provided that one collects data for the dispersion of energy levels over a sufficiently large range of magnetic fields, the terms on both sides of Eq. (1) can be found independently using the same data set. This expression is universal and contains no microscopic or materials parameters.

The second expression we find relates properties of two sets of grains which are equivalent macroscopically except for the value of $\tau_{\text {so }}$. It reads

$$
\left\langle g^{2}\right\rangle=\frac{3 g_{0}^{2}}{2 \pi \hbar} \tau_{\mathrm{so}} \delta+\frac{3}{2 \mu_{B}^{2}} \operatorname{var}\left[\sum_{\sigma=\uparrow, \downarrow}\left(\frac{d \varepsilon_{n \sigma}}{d B}\right)_{B \gg B^{*}}\right]_{\tau_{\mathrm{so}} \rightarrow \infty},
$$

where $g_{0}$ denotes the materials bulk value for the $g$ factor ( $g_{0}=2$ for free electrons). In Eq. (2), $\left\langle g^{2}\right\rangle$ is evaluated in the strong-spin-orbit coupling limit $\left(\tau_{\text {so }} \rightarrow 0\right)$. However, the second term on the right-hand side is evaluated for $\tau_{\text {so }} \rightarrow \infty$ (absence of spin-orbit coupling). For example, one may consider two sets of Al:Au grains, one with no doping and another with moderate doping 1

The two terms on the right-hand side of Eq. (2) come from distinct contributions. The first term, the "spin 
part," is associated with the Debye mechanism of energy dissipation associated with spin reorientations; the second one, the "orbital part," is due to the eddy currents induced in the grain. We note that the spin-orbit scattering rate $1 / \tau_{\text {so }}$ apparently may be estimated for a given material or host-dopant pair ${ }^{6}$ Equation (2) permits us to separate the spin and orbital contributions to the fluctuations of the $g$ factor.

The second moment of the $g$ factor can also be related to the statistics of other spectral quantities, such as the zero-field level curvature

$$
\left\langle g^{2}\right\rangle=\frac{9 \delta}{2 \sqrt{2} \mu_{B}^{2}}\left\langle\left|\left(\frac{d^{2} \varepsilon_{\nu}}{d B^{2}}\right)_{B=0}\right|\right\rangle .
$$

As Eq. (1), this expression is applicable in the strongspin-orbit coupling regime only (symplectic ensemble).

It is important to remark that the $g$ factor in a given metallic nanoparticle is in reality a tensor with three different eigenvalues, even for grains that are statistically isotropic $\stackrel{3.7}{3}$ However, the distribution of the matrix elements of the $g$-factor tensor is still characterized by a single quantity $\left\langle g^{2}\right\rangle$. Throughout our manuscript, when establishing relations between $\left\langle g^{2}\right\rangle$ to other quantities, we consider a magnetic field applied in some fixed but arbitrary direction. Once these relations are established, one may use Ref. 3 to construct the full statistics of the $g$-factor tensor.

We will now establish Eqs. (11)-(31). The main idea behind the derivations is to relate statistical quantities to invariants of the system, such as the traces of the magnetic moment operator. For that purpose, let us begin by writing the Hamiltonian for the disordered (or chaotic) grain in the presence of an applied magnetic field as $\hat{H}(B)=\hat{H}_{0}+B \hat{M}$, where the magnetic moment operator has both orbital and spin parts: $\hat{M}=\hat{M}_{\text {orb }}+\hat{M}_{\text {spin }}$. To simplify the discussion, we assume that the grain is isotropic. We define the $g$ factor of the $n$th energy level as

$$
g_{n} \equiv \frac{1}{2 \mu_{B}}\left|\left(\frac{d \varepsilon_{n \sigma}}{d B}\right)_{B=0}\right|,
$$

where $\left\{\varepsilon_{n \sigma}\right\}$ are the eigenvalues of $\hat{H}_{0}$. Note that due to Kramers degeneracy at $B=0$, the levels are twofold degenerate. We use the index $\sigma$ to distinguish states that are time-reversal conjugate. The matrix elements of $\hat{H}_{0}$ follow either the symplectic $(\beta=4)$ or orthogonal $(\beta=1)$ ensemble statistics, depending on whether spin-orbit coupling is present or absent, respectively. For both cases, the matrix elements of $\hat{M}$, when expressed in the eigenbasis $\{|n \sigma ; 0\rangle\}$ of $\hat{H}_{0}$, fluctuate according to a Gaussian distribution with zero mean. For the symplectic ensemble, the variance of the diagonal matrix elements reads ${ }^{4}$

$$
\left\langle|\langle n \sigma ; 0|\hat{M}| n \sigma ; 0\rangle|^{2}\right\rangle_{\beta=4}=\frac{3 \operatorname{Tr}\left(\hat{M}^{2}\right)}{4 N^{2}},
$$

where the trace runs over the $2 N$ states in the band, with $N \gg 1$ being assumed (the factor of 2 accounts for Kramers degeneracy). The degeneracy at zero field allows us to pick a basis such that $\left\langle n \sigma ; 0|\hat{M}| n \sigma^{\prime} ; 0\right\rangle$ is diagonal in the $\sigma$ indices. Using Eq. (4) and first-order perturbation theory, we find that $\left\langle n \sigma ; 0|\hat{M}| n \sigma^{\prime} ; 0\right\rangle=$ $(-1)^{\sigma} \delta_{\sigma \sigma^{\prime}} g_{n} \mu_{B} / 2$. Thus, from Eq. (5), we arrive at ${ }^{\underline{9}}$

$$
\left\langle g^{2}\right\rangle_{\beta=4}=\frac{3}{\mu_{B}^{2}} \frac{\operatorname{Tr}\left(\hat{M}^{2}\right)}{N^{2}} .
$$

Notice that the quantities on the left-hand side of Eqs. (5) and (6) are defined at $B=0$.

Now we have to write the statistical quantities that appear on the right-hand side of Eqs. (10)-(3), in terms of $\operatorname{Tr}\left(\hat{M}^{2}\right)$. Note that the latter is an invariant and therefore takes the same value at zero or large magnetic fields.

Let us first consider Eq. (1). The variance of the level velocity can be computed in terms of the variance of the matrix elements of the magnetic moment operator since

$$
\left(\frac{d \varepsilon_{\nu}}{d B}\right)_{B=B_{0}}=\left\langle\nu ; B_{0}|\hat{M}| \nu ; B_{0}\right\rangle .
$$

For a sufficiently large magnetic field $B_{0} \gg B^{*}$, timereversal symmetry in the grain is broken. In the presence of strong-spin-orbit scattering, orbital and spin degrees of freedom remain mixed, but the ensemble statistics of the Hamiltonian eigenstates switches from $2 N \times 2 N$ symplectic to $2 N \times 2 N$ unitary $(\beta=2)$. Thus, we need to compute the variance of the matrix elements of the magnetic moment operator in the unitary regime. For this purpose, we make use of the eigenvalues and eigenvectors of the magnetic moment operator: $\hat{M}|k \alpha\rangle=(-1)^{\alpha} M_{k}|k \alpha\rangle$, with $k=1, \ldots, N$ and $\alpha= \pm 1$ due to the time-reversal properties of $\hat{M}: 10$ This yields

$$
\left\langle\nu ; B_{0}|\hat{M}| \nu ; B_{0}\right\rangle=\sum_{k, \alpha}(-1)^{\alpha} M_{k}\left|\left\langle\nu ; B_{0} \mid k \alpha\right\rangle\right|^{2} .
$$

For the unitary ensemble in the large- $N$ limit, the eigenvector amplitudes shown on the right-hand side of Eq. (8) fluctuate independently according to the PorterThomas distribution 11 One finds that $\left.\left|\left\langle\nu ; B_{0} \mid k \alpha\right\rangle\right|^{2}\right\rangle=$ $\frac{1}{2} N$ and $\left\langle\left|\left\langle\nu ; B_{0} \mid k \alpha\right\rangle\right|^{4}\right\rangle=\frac{1}{2} N^{2}$, independently of state indices. Hence, the average matrix element of $\hat{M}$ must vanish and the variance can be written as

$$
\operatorname{var}\left[\left\langle\nu ; B_{0}|\hat{M}| \nu ; B_{0}\right\rangle_{\tau_{\mathrm{so}} \rightarrow 0, \beta=2}\right]=\frac{\operatorname{Tr}\left(\hat{M}^{2}\right)}{4 N^{2}} .
$$

Putting together Eqs. (6), (7), and (9), we arrive at Eq. (11).

To derive Eq. (2), we separate the magnetic moment in terms of spin and orbital parts, $\hat{M}=\hat{M}_{\text {spin }}+\hat{M}_{\text {orb }}$, which are statistically independent from each other. From Eq. (6), we obtain

$$
\left\langle g^{2}\right\rangle_{\beta=4}=\frac{3}{\mu_{B}^{2} N^{2}}\left[\operatorname{Tr}\left(\hat{M}_{\mathrm{spin}}^{2}\right)+\operatorname{Tr}\left(\hat{M}_{\mathrm{orb}}^{2}\right)\right] .
$$


The spin contribution can be written in terms of the imaginary part of the ac spin susceptibility of a free electron gas in the presence of spin-orbit coupling. susceptibility can then be evaluated using conventional means of rate equations at frequencies much larger than the mean level spacing in the grain, yet smaller than the spin-orbit scattering rate. In the limit of $\tau_{\text {so }} \delta / \hbar \ll 1$ one finds 4

$$
\frac{\operatorname{Tr}\left(\hat{M}_{\mathrm{spin}}^{2}\right)}{N^{2}}=\frac{g_{0}^{2} \mu_{B}^{2}}{2 \pi \hbar} \tau_{\mathrm{so}} \delta .
$$

This corresponds to the first term on the right-hand side of Eq. (2).

The orbital contribution in Eq. (10) is ensemble independent and is the same regardless of the presence or absence of the spin-orbit interaction. It is convenient to evaluate it in the limit of $\tau_{\text {so }} \rightarrow \infty$ to decouple spin and orbital degrees of freedom. To relate the $\operatorname{Tr}\left(\hat{M}_{\text {orb }}^{2}\right)$ to the variance of the velocity of spin-resolved levels at large fields, we can use Eq. (17) and note that $\hat{M}_{\text {spin }}$ just induces a constant slope in the dispersion of the energy levels with magnetic field at $\tau_{\text {so }} \rightarrow \infty$, while all fluctuations are caused by $\hat{M}_{\text {orb }}$. Therefore, we write

$$
\sum_{\sigma=\uparrow, \downarrow}\left(\frac{d \varepsilon_{n \sigma}}{d B}\right)_{B=B_{0}}=\left\langle n \uparrow ; B_{0}\left|\hat{M}_{\text {orb }}\right| n \uparrow ; B_{0}\right\rangle
$$

to isolate the fluctuating part (note that $B_{0} \gg B^{*}$ here as well). It is important to observe that in the absence of spin-orbit mixing and for large magnetic fields the statistics of the eigenstate $\left|n \uparrow ; B_{0}\right\rangle$ corresponds to a $N \times N$ unitary ensemble (rather than to $2 N \times 2 N$ when spin and orbital parts are strongly coupled). Similarly to Eq. (8), we can decompose the matrix elements in Eq. (12) using the eigenstates of $\hat{M}_{\text {orb }}$. In this case, however, due to the change in the ensemble dimension, we have $\left\langle\left|\left\langle n \uparrow ; B_{0} \mid k \lambda\right\rangle\right|^{2}\right\rangle=1 / N$ and $\left\langle\left|\left\langle n \uparrow ; B_{0} \mid k \lambda\right\rangle\right|^{4}\right\rangle=2 / N^{2}$ for large $N$, independently of the orbital quantum number $n$. Finally, combining all these results, we arrive at

$$
\operatorname{var}\left[\sum_{\sigma=\uparrow, \downarrow}\left(\frac{d \varepsilon_{n \sigma}}{d B}\right)_{B=B_{0}}\right]_{\tau_{\mathrm{so}} \rightarrow \infty}=\frac{2 \operatorname{Tr}\left(\hat{M}_{\mathrm{orb}}\right)^{2}}{N^{2}} .
$$

Inserting this expression into Eq. (10), we obtain the second term on the right-hand side of (2). We remark that Eq. (2) is fully consistent with Eq. (31) of Ref. 12, where a description of the statistical properties of the $g$ factor including the intermediate crossover regime was developed in terms of phenomenological RMT parameters.

In order to obtain Eq. (3) we follow an approach similar to that employed in the two previous derivations. We define the level curvature at $B=0$ as

$$
K_{n} \equiv\left(\frac{d^{2} \varepsilon_{n \sigma}}{d B^{2}}\right)_{B=0}=2 \sum_{n^{\prime} \neq n} \frac{\sum_{\sigma^{\prime}}\left|\left\langle n \sigma ; 0|\hat{M}| n^{\prime} \sigma^{\prime} ; 0\right\rangle\right|^{2}}{\varepsilon_{n}-\varepsilon_{n^{\prime}}} .
$$

(To simplify the notation, here we set $\varepsilon_{n \sigma}=\varepsilon_{n}$.) Since eigenvalues and eigenfunctions fluctuate independently in the Gaussian ensembles, we find that

$$
\begin{aligned}
\left\langle K_{n}^{2}\right\rangle_{\beta=4}= & \frac{8}{\Delta^{2}}\left[\left\langle\left|\left\langle n \sigma ; 0|\hat{M}| n^{\prime} \sigma^{\prime} ; 0\right\rangle\right|^{4}\right\rangle\right. \\
& \left.+\left\langle\left|\left\langle n \sigma ; 0|\hat{M}| n^{\prime} \sigma^{\prime} ; 0\right\rangle\right|^{2}\right\rangle^{2}\right],
\end{aligned}
$$

with $n \neq n^{\prime}$ and $\sigma, \sigma^{\prime}$ taking arbitrary values. The prefactor in Eq. (15) is defined as

$$
\frac{1}{\Delta^{2}} \equiv 2 \delta \sum_{n} \sum_{n^{\prime} \neq n}\left\langle\frac{\delta\left(\varepsilon_{n}\right)}{\left(\varepsilon_{n}-\varepsilon_{n^{\prime}}\right)^{2}}\right\rangle,
$$

where the delta function is used to fix the energy level in the middle of the band. The average of eigenvalues can be performed using the appropriate two-level cluster function ${ }^{13}$ In the limit $N \rightarrow \infty$, we find

$$
\left.\frac{1}{\Delta^{2}}\right|_{\beta=4}=\frac{\pi^{2}}{9 \delta^{2}},
$$

with $\delta$ denoting the inverse of the mean density of states $\stackrel{14}{\underline{14}}$ The ensemble average of off-diagonal matrix elements of the magnetization is also easily computed in terms of the trace of the magnetization operator in the limit of large $N$ :

$$
\left\langle\left|\left\langle n \sigma ; 0|\hat{M}| n^{\prime} \sigma^{\prime} ; 0\right\rangle\right|^{2}\right\rangle_{n \neq n^{\prime}, \beta=4}=\frac{\operatorname{Tr}\left(\hat{M}^{2}\right)}{4 N^{2}},
$$

and

$$
\left\langle\left|\left\langle n \sigma ; 0|\hat{M}| n^{\prime} \sigma^{\prime} ; 0\right\rangle\right|^{4}\right\rangle_{n \neq m, \beta=4}=\frac{1}{8}\left[\frac{\operatorname{Tr}\left(\hat{M}^{2}\right)}{N^{2}}\right]^{2} .
$$

Inserting Eqs. (17)-(19) into (15) we arrive at

$$
\left\langle K_{n}^{2}\right\rangle_{\beta=4}=\frac{\pi^{2}}{6 \delta}\left[\frac{\operatorname{Tr}\left(\hat{M}^{2}\right)}{N^{2}}\right]^{2} .
$$

For the symplectic ensemble, one can show 15.16 that $\sqrt{\left\langle K_{n}^{2}\right\rangle}=(\pi \sqrt{3} / 4)\left\langle\left|K_{n}\right|\right\rangle$. Thus, using this relation and combining Eqs. (61) and (201), we obtain Eq. (3).

Equation (3), like Eq. (11), also involves only quantities that are directly measurable. However, in practice, the difficulty in obtaining large statistics for the second derivative at $B=0$ from the tunneling conductance data makes it less appealing when applied to experiments $\underline{6.7}$

Once the variance of the level velocity is obtained from the experimental data, it may also allow for another test of RMT. Consider the level velocity correlation function 8,17

$$
\begin{aligned}
C_{\mu}(\Delta B)=\frac{1}{\delta^{2}} & {\left[\left\langle\left(\frac{d \varepsilon_{\nu}}{d B}\right)_{B=B_{0}+\Delta B}\left(\frac{d \varepsilon_{\nu}}{d B}\right)_{B=B_{0}}\right\rangle\right.} \\
& \left.-\left\langle\left(\frac{d \varepsilon_{\nu}}{d B}\right)_{B=B_{0}}\right\rangle^{2}\right] .
\end{aligned}
$$


For a pure ensemble, this correlation function can be rescaled to a universal form. Defining the correlation field as

$$
B_{c} \equiv 1 / \sqrt{C_{\nu}(0)}
$$

and calling $x=\Delta B / B_{c}$ and $c(x)=B_{c}^{2} C_{\nu}(\Delta B)$, the dimensionless correlation function in the unitary ensemble has the asymptotes ${ }^{18}$

$$
c(x)= \begin{cases}1-2 \pi^{2} x^{2}, & x \ll 1, \\ -1 /(\pi x)^{2}, & x \gg 1 .\end{cases}
$$

The full shape of the correlation function is known from numerical simulations,, 8.18 as well as from analytical calculations 19

Finally, it is interesting to note that the correlation field is related to the amount of disorder in the grains when the electron motion is diffusive. It is straightforward to show that at $\tau_{\text {so }} \rightarrow \infty$,

$$
B_{c}=\kappa \frac{\Phi_{0} / L^{2}}{\sqrt{k_{F}^{2} l L}},
$$

where $\Phi_{0}$ is the flux quantum, $L$ is the grain linear size, $k_{F}$ is the Fermi wavelength, and $\kappa$ is a dimensionless coefficient that depends on the grain geometry. For a spherical shape, $\kappa=3 \pi /(2 \sqrt{5})$, in which case $L$ is the grain radius. By measuring the variance of the level velocity at large fields, one can obtain $C_{\nu}(0)$ and find the experimental value of $B_{c}$ from Eq. (22). Using Eq. (24), one can then get an independent estimate of the amount of disorder present in the grain. Another approach is to fit the universal curve ${ }^{8.18} c(x)$ to the experimental data and obtain $C_{\nu}(0)$ as a fitting parameter.

In summary, we have shown that it is possible to relate the second moment of the $g$ factor of metallic nanoparticles with strong spin-orbit coupling to other spectral statistics of energy levels without resorting to any microscopic parameter. Our results also show that it is possible to estimate the spin and orbital contributions to the fluctuations of the $g$ factor by comparing data taken from nanoparticles doped and undoped with a heavy-element metal. We suggest that a fitting of the data to a universal, dimensionless level velocity correlation function may provide an additional test of the applicability of random matrix theory to these systems and allow us to extract information about the intragrain disorder.

This work was supported in part by NSF grants No. DMR 02-37296, No. DMR 04-39026 (L.I.G.) and No. CCF 0523603 (E.R.M.). C.H.L. acknowledges partial support in Brazil from CNPq, Instituto do Milênio de Nanociências, and FAPERJ. E.R.M. acknowledges partial support from the Interdisciplinary Information Science and Technology Laboratory ( $\left.\mathrm{I}^{2} \mathrm{Lab}\right)$ at UCF. We are grateful to Y. Fyodorov, J. Petta, and F. von Oppen for enlightening discussions. L.I.G. and E.R.M. thank the Instituto de Física at UERJ, Brazil, and the Aspen Center for Physics for the hospitality.
1 D. G. Salinas, S. Guéron, D. C. Ralph, C. T. Black, and M. Tinkham, Phys. Rev. B 60, 6137 (1990).

2 D. Davidović and M. Tinkham, Phys. Rev. Lett. 83, 1644 (1999).

3 P. W. Brouwer, X. Waintal, and B. I. Halperin, Phys. Rev. Lett. 85, 369 (2000).

4 K. A. Matveev, L. I. Glazman, and A. I. Larkin, Phys. Rev. Lett. 85, 2789 (2000).

5 R. A. Serota, Solid State. Comm. 117, 605 (2001).

6 J. R. Petta and D. C. Ralph, Phys. Rev. Lett. 87, 266801 (2001).

7 J. R. Petta and D. C. Ralph, Phys. Rev. Lett. 89, 156802 (2002).

8 A. Szafer and B. L. Altshuler, Phys. Rev. Lett. 70, 587 (1993).

9 An analogous relation was derived for the variance of the persistent current of a mesoscopic ring in the presence of spin-orbit coupling by V. E. Kravtsov and M. R. Zirnbauer, Phys. Rev. B 46, 4332 (1992).

10 The magnetic moment operator is antisymmetric with respect to time reversal. Therefore, eigenvectors related by time reversal have eigenvalues equal in amplitude but with opposite signs.

11 C. E. Porter, in Statistical Theories of Spectra: Fluctuations, edited by C. E. Porter (Academic Press, New York, 1965).

12 S. Adam, M. L. Polianski, X. Waintal, and P. W. Brouwer, Phys. Rev. B 66, 195412 (2002).

13 M. L. Mehta, Random Matrices, 3rd edition (Academic Press, Amsterdam, 2004).

14 At $B=0$, due to Kramers degeneracy, $\delta$ is equal to half of the mean level spacing.

15 Y. V. Fyodorov and H.-J. Sommers, Z. Phys. B 99, 123 (1995).

16 F. von Oppen, Phys. Rev. E 51, 2647, (1995).

17 B. D. Simons and B. L. Altshuler, Phys. Rev. Lett. 70, 4063 (1993).

18 B. D. Simons and B. L. Altshuler, Phys. Rev. B 48, 5422 (1993).

19 I. E. Smolyarenko and B. D. Simons, J. Phys. A 36, 3551 (2003). 